\title{
AVALIAÇÃ̃ DOS EFEITOS DO ULTRASSOM TERAPÊUTICO SOBRE A CAFEÍNA E VERIFICAÇÃO DA LIBERAÇÃO EM SISTEMA DE DIFUSÃO VERTICAL
}

\author{
João Alberto Tassinary, Paula Bianchetti e Claudete Rempel \\ Centro de Ciências Biológicas e da Saúde, Centro Universitário UNIVATES, Av. Avelino Tallini, 171, 95900-000 Lajeado - RS, Brasil \\ Simone Stülp* \\ Centro de Ciências Exatas e Tecnológicas, Centro Universitário UNIVATES, Av. Avelino Tallini, 171, 95900-000 Lajeado - RS, Brasil
}

Recebido em 2/12/10; aceito em 30/4/11; publicado na web em 14/6/11

\begin{abstract}
EVALUATION OF EFFECTS TO THE THERAPEUTICAL ULTRASOUND ON CAFFEINE AND THE RELEASE IN VERTICAL DIFFUSION SYSTEMS. Currently, the physiotherapists use the phonoforesis, which consists in the therapeutical ultrasound (US) used to increase the drug molecules migration through the skin, however, the US can shows oxidative effects, and is used, for example, in chemical reactions acceleration. The present study aimed to perform the electrochemical evaluation and the diffusion investigation of gel/caffeine 5\% solutions submitted to therapeutical US (continuous mode, $1.0 \mathrm{~W} \mathrm{~cm}^{-2}$ and $1 \mathrm{MHz}$ ). It this study, it has been verified diffusion increase and a possible oxidation of the caffeine molecules, when subjected to therapeutical US.
\end{abstract}

Keywords: phonoforesis; therapeutical ultrasound; caffeine.

\section{INTRODUÇÃO}

A utilização de ultrassom (US) em diferentes aplicações na área das ciências experimentais tem ganhado destaque, devido ao fato do US poder ser usado como fonte de energia para a ocorrência de reações químicas. ${ }^{1,2} \mathrm{O}$ US apresenta diferentes formas de uso, nas mais variadas frequências, sendo que na área da fisioterapia seu uso ocorre nas frequências de 1 e $3 \mathrm{MHz} .^{3}$

Ainda, no âmbito da fisioterapia, a utilização do US terapêutico pode ocorrer com e sem a conjugação de fármacos, sendo que quando há esta associação, a técnica é denominada fonoforese. ${ }^{4}$ Diversos estudos têm sido realizados avaliando a ação e os efeitos produzidos pela ação do US sobre estes fármacos, ${ }^{5}$ envolvendo, por exemplo, o aumento de permeação destes na pele. ${ }^{6,7}$ Além destas técnicas, ainda na área de atuação da fisioterapia, tem-se outra importante técnica, que igualmente facilita a permeação de fármacos em contato com a pele, a iontoforese ${ }^{8}$ também amplamente estudada.

Na literatura, há estudos relacionados à aplicação de US in vivo, dentre os quais se destaca o de $\mathrm{Hsieh}^{9}$ que, a partir de pesquisas experimentais com a utilização de diclofenaco em ratos, apresentou uma diminuição dos sintomas da artrite nestes animais quando da aplicação da fonoforese, ou seja, US terapêutico associado ao diclofenaco.

Em outros estudos, ${ }^{10}$ foram determinadas as condutividades de 37 meios condutores que são comercialmente utilizados na fonoforese, com a finalidade de facilitar e orientar a escolha desses durante a prática clínica dos fisioterapeutas. O estudo mostrou que, de maneira geral, os produtos na forma de gel apresentaram valores de condutividade elevados. Ainda, os fármacos de aplicação tópica com denominações de gel ou emulgel registraram também valores altos, contrastando, assim, com cremes, loções e pomadas, que apresentaram condutividades baixas, não sendo aconselhável a sua utilização conjuntamente com o US.

Dentre as substâncias que vêm sendo agregadas à onda sônica, a cafeína (Figura 1) possui destaque, sendo caracterizada, ${ }^{11}$ como

*e-mail: stulp@univates.br um pó ou cristais brancos, pouco solúvel em água e podendo ser extraída da Coffea arabica. A cafeína está entre as três metilxantinas de ocorrência natural farmacologicamente ativas e também pode ser encontrada em chás. ${ }^{12}$<smiles>CN1C(=O)C2C(N=CN2C)N(C)C1=O</smiles>

Figura 1. Estrutura química da cafeína

É na natureza que o homem busca, desde primórdios de sua existência, recursos que melhorem sua condição de vida para, assim, aumentar suas chances de sobrevivência pela melhoria de sua saúde. ${ }^{13}$ As plantas medicinais são amplamente pesquisadas, tanto farmacologicamente quanto para o desenvolvimento de novas drogas e produtos tópicos, não somente quando os seus constituintes são usados diretamente como agentes terapêuticos, mas também como matérias-primas para a síntese, ou modelos para compostos farmacologicamente ativos. ${ }^{14}$

Assim as plantas medicinais, as preparações fitofarmacêuticas e os produtos naturais isolados, como a cafeína, são pesquisados e utilizados em todo o mundo e, desta forma, representam um mercado que movimenta bilhões de dólares, tanto em países industrializados como em desenvolvimento. ${ }^{15}$

A cafeína, como princípio ativo dermatológico, exerce ação no tecido adiposo subcutâneo, através da inibição da fosfodiesterase, tendo um efeito lipolítico. Entretanto, para que a molécula transponha a principal barreira à penetração cutânea deve ser associada a promotores de absorção cutânea ou veiculada através de lipossomas. ${ }^{16}$ Segundo alguns autore ${ }^{17}$ a cafeína também ocasiona uma redução da espessura da hipoderme devido ao achatamento nos lóbulos de tecido adiposo.

Pesquisas in vitro, utilizando células de difusão vertical, ${ }^{7}$ sugerem que o US acentua e acelera significativamente a permeação da 
cafeína através da pele, permitindo a aplicação da fonoforese, não ocasionando alterações na pele exposta ao US, sendo o estudo realizado por meio de análises histológicas e de microscopia eletrônica.

É fundamental ressaltar que a promoção de permeação de fármacos pela pele através da onda sônica pode levar a maior eficácia da liberação de ativos; aumento do fluxo ou retenção do mesmo, aumento da liberação localizada; tópica ou dos tecidos alvo através da pele; ou, ainda, a combinação das hipóteses citadas. No entanto, esta liberação poderá promover efeitos tóxicos tanto intracelulares como extracelulares, caso não haja utilização adequada da quantidade do composto associado, ${ }^{18} \mathrm{ou}$, ainda, devido à ocorrência de alteração da estrutura química do composto. ${ }^{5}$ Poderão ocorrer efeitos adversos e não desejáveis ao tratamento, assim como a utilização adequada poderá promover redução de possíveis agentes tóxicos ao organismo. Determinações eletroquímicas podem auxiliar na avaliação de possíveis degradações de princípios ativos, além de possibilitar a determinação da toxicidade dos mesmos. ${ }^{19,20}$

Diante do exposto, o objetivo do presente trabalho foi realizar estudos da liberação da cafeína, quando adicionada a um gel transmissor, com e sem a aplicação do US terapêutico, além de avaliar, por meio eletroquímico, possíveis reações de oxidação ocorridas após a aplicação deste. A importância deste trabalho reside na avaliação da promoção, por parte do US terapêutico, do aumento de liberação (facilitador) do fármaco para o meio, em ensaios in vitro, investigando possíveis alterações estruturais ocorridas neste sistema.

\section{PARTE EXPERIMENTAL}

\section{Reagentes e preparo das amostras}

Nos ensaios de incidência de onda sônica, as amostras utilizadas para a análise foram obtidas a partir de gel condutor ${ }^{21}$ da marca Mercur (hidrogel) com fabricação em 13/09/08 e validade até 09/2011, lote 237 506-403, com adição de cafeína a 5\%, produzido pelo laboratório Sigma-Aldrich, lote 029k 1023, com data de fabricação em 31/08/2009 e com validade até 14/09/2014. As amostras de cafeína foram preparadas a partir de gel condutor e cafeína na concentração de 5\%. Esta concentração de cafeína foi utilizada por ser a comumente encontrada em géis comerciais para aplicações fisioterapêuticas. Para garantir a homogeneidade da amostra utilizou-se um agitador mecânico da marca Fisatom modelo 703 D.

\section{Aplicação do ultrassom terapêutico in vitro}

Foi montado um sistema sobre uma mesa agitadora (Figura 2) da marca Tecnal modelo TE - 140, que foi ajustada em $23 \mathrm{rpm},{ }^{22} \mathrm{com}$ a finalidade de simular a aplicação in vivo sem a formação de ondas estacionárias, que ocorrem como resultado da sobreposição das ondas

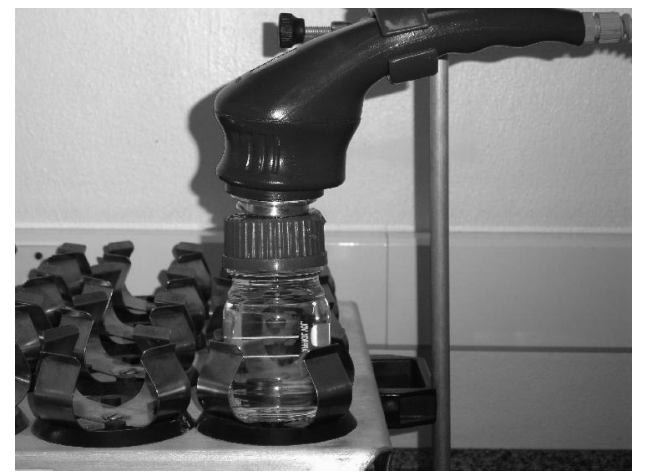

Figura 2. Sistema para aplicação do ultrassom terapêutico in vitro refletidas com as ondas incidentes ${ }^{23}$ sendo necessária a movimentação contínua do transdutor. Acoplada à mesa agitadora estava uma célula receptora contendo água/álcool etílico 1:1 (solução receptora), em contato com uma membrana e esta com o gel contendo o fármaco, e o transdutor de US.

No acompanhamento deste experimento in vitro foram analisados parâmetros eletroquímicos, a partir da técnica de voltametria cíclica, onde se utilizou uma célula convencional de três eletrodos, com o auxílio de um potenciostato modelo PGSTAT128N da marca Autolab/ Ecochemie, com velocidade de varredura de $20 \mathrm{mV} \mathrm{s}^{-1}$. A célula eletroquímica continha um eletrodo de trabalho de platina com área de $0,385 \mathrm{~cm}^{2}$, um contraeletrodo de platina e um eletrodo de referência sendo constituído de um fio de prata revestido com cloreto de prata. Todos os experimentos eletroquímicos foram realizados em triplicata e apresentaram repetibilidade entre os diferentes experimentos, sendo que os dados apresentados são médias das triplicatas realizadas. Os gráficos foram obtidos no software Origin ${ }^{\circledR}$. A determinação do percentual de cafeína oxidada foi feita por espectrofotometria UV/ Vis (Cary 100 Bio).

\section{Análise de liberação da cafeína em sistema de difusão vertical}

Os experimentos de liberação foram realizados em uma célula de difusão vertical, contendo uma membrana de acetato de celulose (Sartorius Stedim com porosidade de $0,45 \mu \mathrm{m}$ ), demonstrada na Figura 3. Preparou-se o sistema, sobre uma chapa agitadora (Velp Scientifica), contendo uma célula de camisa dupla de volume $200 \mathrm{~mL}$ da marca Germany, contendo água aquecida a $37{ }^{\circ} \mathrm{C}$ por um banho ultratermostatizado (MA-184 Marconi), simulando a temperatura corporal; dentro desta foi inserida a célula de difusão (100 mL) com solução receptora de água e álcool etílico 99,5\% (Nuclear) 1:1 e uma barra agitadora magnética para manter a homogeneidade da amostra. Em contato com a área exposta da membrana de acetato de celulose foi adicionado o agente acoplador (hidrogel) e acima o transdutor do US. ${ }^{24} \mathrm{~A}$ aplicação do US terapêutico foi realizada com o auxílio de um equipamento da marca DGM Eletrônica Ltda. e foi aplicado em cada uma das amostras analisadas, na seguinte modulação: modo contínuo, $1 \mathrm{MHz}$, intensidade de $1,0 \mathrm{~W} \mathrm{~cm}^{-2}$ e tempo de $10 \mathrm{~min}$ de aplicação, obtido através do cálculo área de aplicação/era do transdutor. Após,

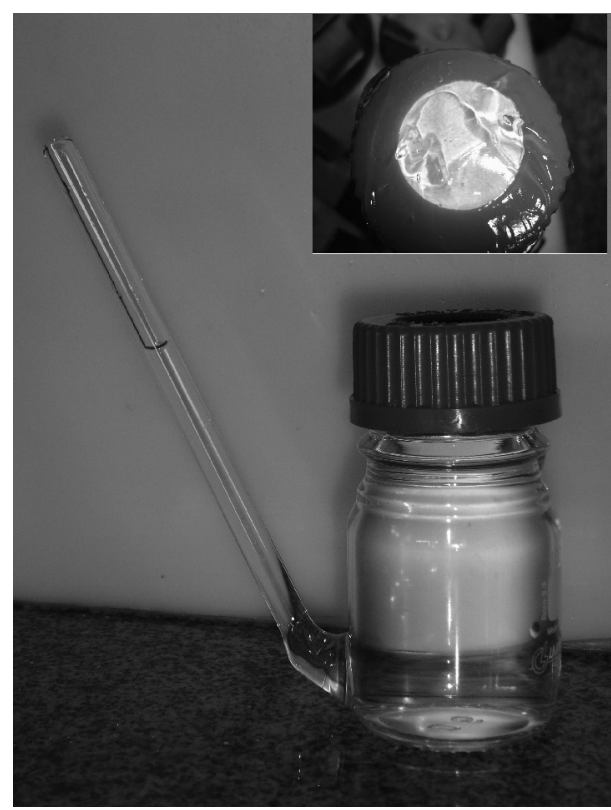

Figura 3. Célula de difusão utilizada nos ensaios de liberação, no detalhe, área exposta para aplicação do US sobre membrana de acetato de celulose 
foram realizadas análises de varreduras espectrofotométricas (195 a 990 nm) de alíquotas retiradas da célula de difusão, para a verificação da presença do substrato na solução receptora. Os ensaios foram obtidos através de um espectrofotômetro Cary 100 Bio UV/Vis. Todas as análises foram realizadas em triplicata.

\section{RESULTADOS E DISCUSSÃO}

\section{Análise de voltametria cíclica}

A partir do sistema que simulou a aplicação in vivo, montado sobre a mesa agitadora, foram realizados os ensaios de caracterização eletroquímica, por meio da utilização da técnica de voltametria cíclica. Estes experimentos foram realizados em amostras de hidrogel e hidrogel + cafeína 5\%, antes e após a aplicação de US terapêutico, por $10 \mathrm{~min}$, com velocidade de varredura de $20 \mathrm{mV} \mathrm{s}^{-1}$, sendo que os potenciais são referenciados em relação ao eletrodo de $\mathrm{Ag} / \mathrm{AgCl}$. $\mathrm{Na}$ Figura 4, tem-se um voltamograma cíclico do hidrogel antes e após a aplicação de US por $10 \mathrm{~min}$.

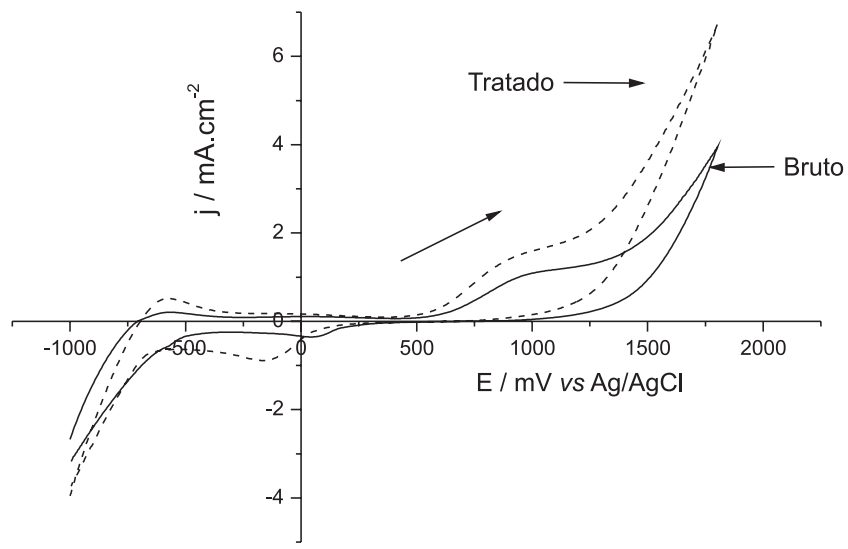

Figura 4. Voltamograma cíclico do hidrogel antes e após a aplicação de ultrassom (10 $\mathrm{min}), v=20 \mathrm{mV} \mathrm{s}^{-1}$

Avaliando a Figura 4, verifica-se que houve alteração eletroquímica no sistema, após exposição por $10 \mathrm{~min}$ ao US terapêutico, pela visualização da alteração da área do voltamograma. Confirmando a análise, o cálculo da carga evidencia um acréscimo de $\mathrm{Q}=5,291 \mathrm{x}$ $10^{-2} \mathrm{C} \mathrm{cm}^{-2}$ para $\mathrm{Q}=9,991 \times 10^{-2} \mathrm{C} \mathrm{cm}^{-2}$, do hidrogel bruto para $\mathrm{o}$ tratado, respectivamente.

Além da alteração nos valores de carga, há a presença de um potencial de pico $\left(\mathrm{E}_{\mathrm{p}}\right)$ em valores próximos a $0,9 \mathrm{~V}$, sendo que sistemas contendo compostos orgânicos normalmente apresentam picos de oxidação nesta região ${ }^{25-27}$ e o hidrogel estudado contém em sua formulação carbopol (polímero vinílico) e propilenoglicol, compostos orgânicos amplamente utilizados em formulações dermatológicas. ${ }^{21,28,29}$

$\mathrm{Na}$ Figura 5, tem-se o voltamograma cíclico característico do sistema hidrogel + cafeína $5 \%$, com velocidade de varredura de $20 \mathrm{mV} \mathrm{s}^{-1}$, com e sem a aplicação do US terapêutico.

Avaliando a Figura 5, verifica-se que houve alteração eletroquímica do sistema hidrogel/cafeína, após exposição por 10 min ao US terapêutico, em função da área do voltamograma, sendo acrescida após a aplicação da onda sônica. Em termos de valores de carga, há um acréscimo de $\mathrm{Q}=8,080 \times 10^{-2} \mathrm{C} \mathrm{cm}^{-2}$ para $\mathrm{Q}=1,005 \times 10^{-1} \mathrm{C}$ $\mathrm{cm}^{-2}$, do sistema hidrogel/cafeína bruta para a tratada, respectivamente, sendo este de uma ordem de grandeza. Os resultados obtidos (voltamogramas) neste estudo são semelhantes aos obtidos em outro estudo de determinação eletroquímica de cafeína, ${ }^{30}$ porém neste caso com eletrodos de pasta de carbono e a cafeína em solução aquosa.

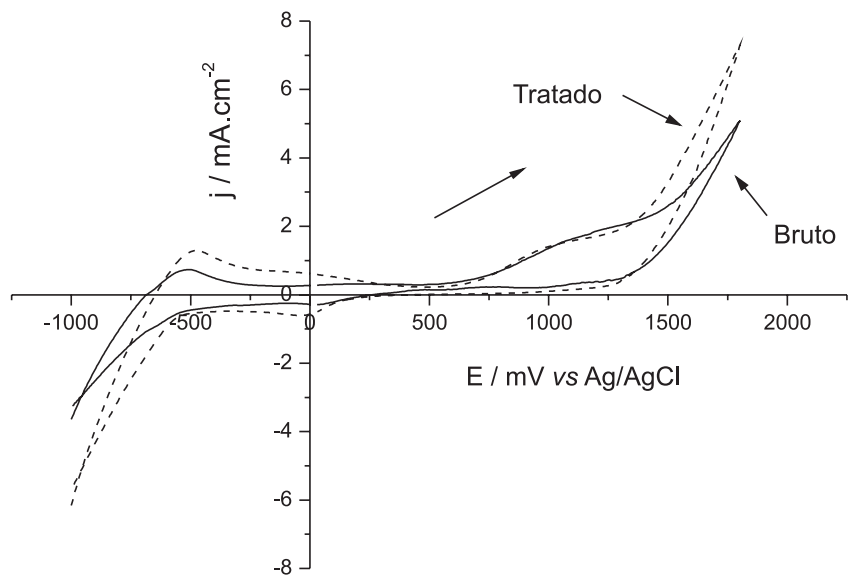

Figura 5. Voltamograma cíclico do sistema hidrogel/cafeína antes e após a aplicação de ultrassom (10 $\mathrm{min}), v=20 \mathrm{mV} \mathrm{s}^{-1}$

Esse aumento nos valores de carga, após exposição ao US terapêutico pode evidenciar uma maior susceptibilidade à oxidação do sistema após a aplicação do US terapêutico. 5

Além deste efeito, avaliando-se a Figura 5, verifica-se que em potenciais próximos a $1,1 \mathrm{~V}$, tem-se um pico anódico, com corrente de pico $\left(\mathrm{I}_{\mathrm{p}}\right)$ da ordem de $610 \mu \mathrm{A}$, sendo que esta onda é no potencial eletroquímico característico da cafeína. ${ }^{30,31} \mathrm{Em}$ trabalhos publicados envolvendo a determinação voltamétrica da cafeína, os autores encontraram também picos de oxidação nesta região de potenciais e verificaram que, dependendo do eletrodo de trabalho utilizado, há pequenos deslocamentos nos potenciais medidos. ${ }^{31-34}$ Além do efeito do eletrodo de trabalho, outro aspecto importante é o valor de $\mathrm{pH}$ das amostras analisadas, sendo que este pode também influenciar nos deslocamentos dos potenciais de pico $\left(\mathrm{E}_{\mathrm{p}}\right)$; avaliando-se a Figura 5, verifica-se um pequeno deslocamento quando comparadas as amostras com e sem submissão à onda sônica, sendo que o pH do sistema após incidência de radiação também tende a uma acidificação.

Ainda segundo alguns trabalhos envolvendo o estudo de soluções contendo cafeína, ${ }^{30,32}$ o mecanismo proposto de eletro-oxidação (Figura 6) envolve 4 elétrons e 4 prótons e ocorre em duas etapas. A primeira etapa envolve a perda de 2 elétrons e 2 prótons, ocorrendo a oxidação da molécula no carbono 8 e nitrogênio 9 , obtendo-se assim um ácido úrico substituído (composto intermediário). Este composto intermediário é oxidado em uma segunda etapa que envolve a perda de mais 2 elétrons, formando então um análogo ao ácido úrico, dihidroxilado nas posições 4 e 5 . Esta molécula, segundo a literatura, ${ }^{32}$ é rapidamente fragmentada. Desta forma, após sua fragmentação, provavelmente há a formação de ureia substituída.<smiles>CN1C=NC2C1C(=O)N(C)N(C)C(=O)N2C</smiles>

Figura 6. Mecanismo de eletro-oxidação da cafeína

Aplicando critérios de diagnóstico para o sistema estudado como, por exemplo, a determinação do número de elétrons envolvidos a partir do valor de carga $\mathrm{Q}$ sob o pico voltamétrico e da corrente de pico $\left(I_{p}\right),{ }^{35,36}$ tem-se que o número de elétrons a partir dos voltamogramas cíclicos analisados é próximo a 4, para a reação de oxidação da cafeína, no potencial de pico de $1,1 \mathrm{~V}$, confirmando os resultados obtidos na literatura.

Além disso, verifica-se que o tratamento com US terapêutico 
promove um deslocamento da reação de desprendimento de oxigênio (RDO) para valores de potenciais mais negativos, no sistema hidrogel e hidrogel + cafeína após a aplicação do US terapêutico, indicando que a oxidação do sistema é facilitada após a aplicação do US, pelas características da onda sônica. ${ }^{1}$ Cabe destacar que o ultrassom promove efeitos térmicos e não térmicos quando da sua aplicação. Dentre estes efeitos, destaca-se a corrente acústica, ou cavitação, ${ }^{37}$ que promove mudanças no arranjo da camada córnea da epiderme quando da aplicação terapêutica in vivo do US. Este é o principal mecanismo para o fonoforese, ${ }^{4}$ pela indução de desordem na bicamada lipídica da camada córnea, aumentando o transporte através da membrana, e este efeito sobre os tecidos alvo se deve ao efeito tixotrópico, facilitando assim a absorção de fármacos. A cavitação tem por consequência a formação de cavidades ${ }^{1}$ com dimensões de micrômetros em fluidos contendo gases. Tal fenômeno pode, inclusive, ocasionar a alteração de interações intermoleculares, bem como ligações duplas também podem ser afetadas pela agitação do sistema. ${ }^{38}$

Após a realização dos experimentos voltamétricos, foram realizadas medidas espectrofotométricas, da solução antes e após o tratamento com ultrassom terapêutico, e determinada a concentração de cafeína na amostra, em comprimento de onda de $272 \mathrm{~nm} .^{31}$ Desta forma, verificou-se que em torno de $11 \%$ da cafeína contida na amostra foi oxidada.

\section{Análises espectrofotométricas}

O gráfico apresentado na Figura 7 foi obtido a partir da análise da absorbância das diferentes amostras de hidrogel e hidrogel + cafeína 5\%, com e sem a aplicação do US, nos ensaios de liberação com membrana de acetato de celulose, através de varredura em toda a faixa espectral (195-990 nm), sendo considerados os valores próximos a $280 \mathrm{~nm}$ como a região de absorbância máxima.

Segundo a literatura, ${ }^{39}$ alguns componentes do café verde (cru), tais como proteínas, peptídeos, aminoácidos aromáticos livres, trigonelina e cafeína, apresentam absorção em $280 \mathrm{~nm}$. Porém, a análise da cafeína no comprimento de onda específico de $272 \mathrm{~nm}$ aumenta a seletividade do método e, portanto, sua precisão e exatidão. ${ }^{26}$

$\mathrm{Na}$ Figura 7 tem-se espectro UV/Vis das amostras de hidrogel e hidrogel + cafeína 5\%, com e sem exposição ao US terapêutico no modo contínuo.

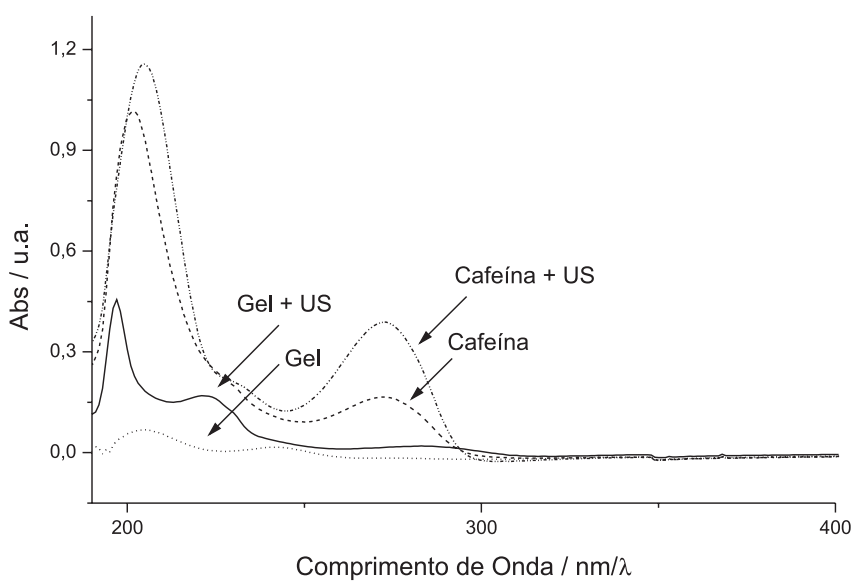

Figura 7. Espectro UV/Vis das amostras de hidrogel e hidrogel + cafeína 5\%, com e sem exposição ao US terapêutico no modo contínuo

A Figura 7 permite a visualização da diferença de absorbância entre as amostras, sendo que o hidrogel e o hidrogel após aplicação de US não apresentaram incremento da banda $(\lambda=272 \mathrm{~nm})$, já o sistema hidrogel + cafeína mostrou uma discreta absorbância por volta da faixa de 0,2 (de absorbância) e quando este foi associado ao tratamento com US terapêutico revelou um maior incremento da banda na região de absorbância característico da cafeína por volta dos $272 \mathrm{~nm}$, sugerindo então uma liberação mais acentuada do ativo para o meio, provavelmente por conta da ação física da onda sônica, com valores de absorbância por volta de 0,4 . Em termos de concentração, a partir de curva de calibração realizada com soluções de cafeína em solução água/álcool 1:1, tem-se que, antes da aplicação de US, a concentração de cafeína na solução receptora é de 5,25 x 10 $0^{-5}$ mol L $\mathrm{L}^{-1}$, enquanto que após a aplicação de US terapêutico, ocorre a liberação de $1,73 \times 10^{-4} \mathrm{~mol} \mathrm{~L}^{-1}$ de cafeína, sendo o aumento de uma ordem de grandeza, estando estes valores abaixo dos níveis tóxicos, em termos plasmáticos, que são da ordem de concentrações milimolares. ${ }^{40,41}$ Ou seja, o gráfico evidenciou incrementos de absorbância nas bandas características da cafeína, sugerindo uma maior liberação do ativo quando associado à onda sônica terapêutica, portanto, o US se apresentou como facilitador no processo de liberação da cafeína.

Diante dos resultados obtidos, percebe-se que há a ocorrência de um aumento de liberação de cafeína para o sistema receptor, devido à aplicação do US terapêutico, sendo este um aspecto positivo para aplicações fisioterapêuticas, porém, há o indicativo de oxidação, ou seja, alteração da estrutura da molécula de cafeína, sendo este um aspecto negativo, já que a aplicação da cafeína, em termos terapêuticos, ocorre devido às características que a molécula possui em função de sua estrutura química. Estudos posteriores são necessários para verificar se a atividade da cafeína é mantida após a ação da onda sônica como, por exemplo, a atividade anti-inflamatória.

\section{CONCLUSÃO}

Os resultados obtidos elucidaram a possibilidade de detecção da degradação da cafeína através da técnica de voltametria cíclica. Sugere-se então que a ação física do US, no modo contínuo, tem a capacidade de oxidar os sistemas analisados. As amostras de hidrogel e hidrogel + cafeína apresentaram um deslocamento da reação de desprendimento de oxigênio (RDO), apresentando também pico característico de sistemas que contêm cafeína, em potenciais em torno de $1,1 \mathrm{~V}$.

Considerando que a onda sônica terapêutica associada a agentes com princípios ativos é uma técnica diariamente elencada na prática clínica fisioterapêutica e sabendo-se que houve uma degradação dos sistemas estudados, após a aplicação da técnica de fonoforese por 10 min no modo contínuo, torna-se fundamental o desenvolvimento de um conhecimento aprofundado de seus efeitos ao interagir com tecidos, ou seja, a ação anti-inflamatória e lipolítica dos ativos estudados, cientificamente comprovadas, podem ser discutidas, uma vez que a onda sônica pode ocasionar a alteração da estrutura química dos ativos.

Outro fator relevante na prática clínica é a permeação dos ativos nos tecidos superficiais; como fase inicial para tal análise identificouse a capacidade de liberação das substâncias. Os resultados sugerem uma maior liberação da cafeína quando associada à onda sônica terapêutica, ou seja, o US se apresentou como facilitador no processo de liberação da cafeína para o meio.

\section{REFERÊNCIAS}

1. Garbellini, G. S.; Salazar-Banda, G. R.; Avaca, L. A.; Quim. Nova 2008, $31,123$.

2. Martines, M. U.; Davolos, M. R.; Jafelicci Júnior, M.; Quim. Nova 2000, 23, 251.

3. Matheus, J. P. C.; Oliveira, F. B.; Gomide, L. B.; Milani, J. G. P. O.; Volpon, J. B.; Shimano, A. C.; Rev. Bras. Fisioter. 2008, 12, 241. 
4. Mitragotri, S.; Kost, J.; Adv. Drug Delivery Rev. 2004, 56, 589.

5. Bianchetti, P.; Tassinary, J. A. F.; Cerutti, D. G. U.; Barnes, D.; Ethur, E. M.; Stülp, S.; Revista Liberato 2009, 10, 139.

6. Mutoh, M.; Ueda, H.; Nakamura, Y.; Hirayama, K.; Atobec, M.; Kobayashia, D.; Morimoto, Y.; J. Controlled Release 2003, 92, 137.

7. Boucaud, A.; Machet, L.; Arbeille, B.; Machet, M. C.; Sournac, M.; Mavon, A.; Patat, F.; Vaillant, L.; Int. J. Pharm. 2001, 228, 69.

8. Gratieri, T.; Gelfuso, G. M.; Lopez, R. F. V.; Quim. Nova 2008, 31, 1490.

9. Hsieh, Y. L.; Phys Ther. 2006, 86, 39.

10. Ferreira, L.; Stattmiler, C.; Belo, F.; Pinheiro, H.; Pedrosa, L.; Gaspar, P.; Coutinho, I.; Dias, N.; Arquivos de Fisioterapia 2006, 1, 14.

11. Korolkovas, A.; Química farmacêutica, Guanabara Koogan: Rio de Janeiro, 1988.

12. Rang, H. P.; Farmacologia, $4^{\mathrm{a}}$ ed., Guanabara Koogan: Rio de Janeiro, 2001.

13. http://bvsms.saude.gov.br/bvs/publicacoes/fitoterapia_no_sus.pdf, acessada em Abril 2011.

14. Who; Guidelines on Good Agricultural and Collection Practices (GACP) for Medicinal Plants, $1^{\text {st }}$ ed., World Health Organization: Geneve, 2003.

15. Calixto, J. B. Em Plantas medicinais sob a ótica da Química Medicinal Moderna; Yunes, R. A.; Calixto, J. B., eds.; Argos: Chapecó, 2001, cap. 7.

16. Frézard, F.; Schettini, D. A.; Rocha, O. G. F.; Demicheli, C.; Quim. Nova $\mathbf{2 0 0 5}, 28,511$.

17. Chorilli, M.; Carvalho, L. S.; Pires-de-Campos, M. S. M.; Leonardi, G. R.; Ribeiro, M. C. A. P.; Polacow, M. L. O.; Acta Farm. Bonaerense $\mathbf{2 0 0 5}, 24,14$

18. Rogero, S. O.; Lugão, A. B.; Ikeda, T. I.; Cruz, A. S.; Mat. Res. 2003, 6, 317.

19. Oliveira-Brett, A. M.; Goulart, M. O. F.; Abreu, F. C.; Bioelectrochemistry 2002, 56, 53.

20. Ferreira, S. B.; Gonzaga, D. T. G.; Santos, W. C.; Araújo, K. G. L.; Ferreira, V. F.; Rev. Virtual Quim. 2010, 2, 140.

21. Bolfe, V. J.; Guirro, R. R. J.; Rev Bras Fisioter. 2009, 13, 499.
22. Kramer, J. F.; Arch. Phys. Med. Rehabil. 1984, 65, 223.

23. Low, J.; Reed, A.; Eletroterapia explicada: princípios e prática, $3^{\mathrm{a}}$ ed., Manole: Barueri, 2001.

24. Mendonça, C. C.; Silva, I. C. L.; Rodrigues, K. A.; Campos, M. A. L.; Medeiros, M. C. M.; Casteli, V. C.; Ferrari, M.; Musis, C. R.; Machado, S. R. P.; Rev. Ciênc. Farm. Básica Apl. 2009, 30, 26.

25. Lavagnoli, L. V.; Navarro, M.; Giovani, W. F.; Romero, J. R.; Quim. Nova 1995, 18, 157.

26. Profeti, L. P. R.; Profeti, D.; Olivi, P.; Ecl. Quím. 2005, 30, 75.

27. Noel, M.; Vasu, K. I.; Cyclic Voltammetry and the Frontiers of Electrochemistry, $4^{\text {th }}$ ed., Aspect Publications Ltd.: London, 1990.

28. Martins, M. R. F. M.; Veiga, F.; Rev. Bras. Cienc. Farm. 2002, 38, 33.

29. Baby, A. R.; Filho, C. A. H.; Sarruf, F. D.; Júnior, C. R. T.; Pinto, C. A. S. O.; Zague, V.; Arêas, E. P. G.; Kaneko, T. M.; Velasco, M. V. R.; Braz. J. Pharm. Sci. 2008, 44, 233.

30. Sanghavi, B. J.; Srivastava, A. K.; Electrochim. Acta 2010, 55, 8638.

31. de Maria, C. A. B.; de Moreira, R. F. A.; Quim. Nova 2007, $30,99$.

32. Lourenção, B. C.; Medeiros, R. A.; Rocha-Filho, R. C.; Mazo, L. H.; Fatibello-Filho, O.; Talanta 2009, 78, 748.

33. Zen, J.-M.; Ting, Y.-S.; Anal. Chim. Acta 1997, 342, 175.

34. Aklilu, M.; Tessema, M.; Redi-Abshiro, M.; Talanta 2008, 76, 742.

35. Bard, A. J.; Faulkner, L. R.; Electrochemical methods: Fundamentals and applications, $2^{\text {nd }}$ ed., John Wiley \& Sons: Hoboken, 2000.

36. Chaves, J. A. P.; Araújo, M. F. A.; Varela Júnior, J. J. G.; Tanaka, A. A.; Ecl. Quím. 2003, 28, 9

37. Koeke, P. U.; Parizotto, N. A.; Carrinho, P. M.; Salete, A. C. B.; Ultrasound Med Biol. 2005, 31, 345.

38. Péres, V. F.; Saffi, J.; Melecchi, M. I. S.; Abad, F. C.; Jacques, R. A.; Martinez, M. M.; Oliveira, E. C.; Caramão, E. B.; J. Chromatogr., A 2006, 1105, 115.

39. Santos, M. H.; Batista, B. L.; Duarte, S. M. S.; Abreu, C. M. P.; Gouvêa, C. M. C. P.; Quim. Nova 2007, 30, 604.

40. Garrett; B. E.; Griffiths, R. R.; Pharmacol., Biochem. Behav. 1997, 57, 533.

41. Kerrigan, S.; Lindsey, T; Forensic Sci. Int. 2005, 153, 67. 